\title{
Diagnosis of peri-prosthetic loosening of total hip and knee arthroplasty using ${ }^{18}$ F-Fluoride PET/CT
}

\author{
Sebastian Koob ${ }^{1}$, Florian C. Gaertner ${ }^{2}$, Tom Rainer Jansen ${ }^{1}$, Jan Schmolders ${ }^{1}$, Sascha \\ Gravius $^{1}$, Holger Strunk ${ }^{3}$, Dieter Christian Wirtz ${ }^{1}$ and Markus Essler ${ }^{2}$ \\ ${ }^{1}$ Clinic for Orthopedics and Trauma Surgery, University of Bonn, Bonn, Germany \\ ${ }^{2}$ Clinic for Nuclear Medicine, University of Bonn, Bonn, Germany \\ ${ }^{3}$ Department of Radiology, University of Bonn, Bonn, Germany \\ Correspondence to: Sebastian Koob, email: sebastian.koob@ukbonn.de \\ Keywords: periprosthetic loosening; bone imaging; total hip arthroplasty; periprosthetic infection; ${ }^{18}$ F-Flouride PET/CT \\ Received: October 11, $2018 \quad$ Accepted: February 01, $2019 \quad$ Published: March 15, 2019 \\ Copyright: Koob et al. This is an open-access article distributed under the terms of the Creative Commons Attribution License 3.0 \\ (CC BY 3.0), which permits unrestricted use, distribution, and reproduction in any medium, provided the original author and source \\ are credited.
}

\section{ABSTRACT}

Periprosthetic loosening, either aseptic or induced by periprosthetic joint infection remains a major long term complication and challenge in orthopedics and trauma surgery. Sensitivity of potential loosening of the material and other causes of a painful prosthesis is essential for choosing the respective treatment option and providing the needed resources. ${ }^{18} \mathrm{~F}$-Fluoride is a radiopharmaceutical which shows a high affinity to bone and a rapid blood clearance.

The objective of this study was to assess ${ }^{18} \mathrm{~F}-\mathrm{Fluoride} \mathrm{PET} / \mathrm{CT}$ 's sensitivity and specificity in diagnosing periprosthetic loosening in total hip and knee arthroplasty.

We included 26 patients with 24 hip and 13 knee prostheses in our retrospective study with radiological or clinical suspicion of peri-prosthetic loosening at least one year after implantation. Results of ${ }^{18} \mathrm{~F}-\mathrm{Fluoride} \mathrm{PET} / \mathrm{CT}$ imaging were compared with surgical results or clinical follow-up if surgery was not performed. On the basis of our data we found a sensitivity of $95.00 \%$, a specificity of $87.04 \%$ and an accuracy of $89.19 \%$ for ${ }^{18}$ F-Fluoride PET/CT.

The results of our study show that ${ }^{18} \mathrm{~F}$-Fluoride PET/CT is a useful and promising technique in diagnosing periprosthetic loosening of total hip and knee arthroplasties. Further investigation should focus on different uptake patterns of the isotope in periprosthetic joint infection and therefore distinguishing aseptic from septic loosening and enhancing the diagnostic value of this imaging method.

\section{INTRODUCTION}

Total hip and knee arthroplasties in end-stage arthritis contribute an essential number to today's orthopedic procedures. Periprosthetic loosening, either aseptic or induced by periprosthetic joint infection remains a major long term complication and challenge in orthopedics and trauma surgery. According to estimations between 0.4 and $4 \%$ of joint replacements will face periprosthetic infection and 2-18\% aseptic loosening [1]. When dealing with painful prostheses an early distinguishment between potential loosening of the material and other causes is essential for choosing the respective treatment option and providing the needed resources [2].

According to current understanding a chronic inflammatory process of the bone- prosthesis or cementprosthesis interface causes implant loosening. Wear particles are being phagocytosed by macrophages which induce chronic inflammation and osteolysis with activation of osteoblastic and osteoclastic activity [3, 4]. Early before the gross implant motion sets in and radiographic changes can be recognized, so called micromotion already causes pain and makes diagnosis difficult. Today's standard 
diagnostic procedures for ruling out prosthetic loosening most often include x-ray and bone scan imaging, as well as joint punctures and laboratory tests which feature wide ranges in specificity and sensitivity [1]. Especially plain radiographs often show signs of loosening only after a long delay of time. Traditional bone scan imaging comes along with a low spatial resolution.

${ }^{18}$ F-Fluoride PET/CT provides a promising alternative. ${ }^{18} \mathrm{~F}$-Fluoride is a radiopharmaceutical which shows a high affinity to bone and a rapid blood clearance with high bone-to-background ratio in a shorter time than for standard ${ }^{99 \mathrm{~m}} \mathrm{Tc}$-based tracers $[5,6]$. In combination with positron emission tomography and its excellent spatial resolution ${ }^{18} \mathrm{~F}$-Fluoride PET/CT may offer highly valuable images for detecting loose components of total hip and knee arthroplasties.

Therefore, the objective of this study was to assess ${ }^{18} \mathrm{~F}$-Fluoride $\mathrm{PET} / \mathrm{CT}^{\prime} \mathrm{s}$ sensitivity and specificity in diagnosing periprosthetic loosening in total hip and knee arthroplasty.

\section{RESULTS}

The collective of 26 patients included 15 male and 11 female patients with a mean age of 67.5 years $(50.1$ 92.2 years). We evaluated a total of 24 hip and 13 knee prostheses. Since the high resolution of the imaging allows for a separate evaluation of the femoral, tibial and acetabular part of an arthroplasty we were able to analyze 74 prosthetic components. The mean time between implantation and ${ }^{18} \mathrm{~F}$-Fluoride PET/CT scan was 6.5 years (range $1.0-19.6$ years).

The clinically silent additional 11 prostheses (22 components) were counted as true negative since the ${ }^{18}$ F-Fluoride PET/CT showed no signs of loosening.

For 18 patients and therefore 18 prostheses (36 components, $48.6 \%$ ) the ${ }^{18} \mathrm{~F}$-Fluoride $\mathrm{PET} / \mathrm{CT}$ diagnosis was confirmed or disproved by intraoperative findings. In these cases the surgeon declared the respective components as loose or stable as stated in his operational report.

For 19 prostheses (38 components, 51.4\%) without indication for revision surgery a clinical follow up lasting at least 6 months confirmed the ${ }^{18} \mathrm{~F}$-Fluoride PET/CT diagnosis of a loosened or non loosened prosthesis.

One patient did not undergo revision surgery of two total knee arthroplasties due to age and the high risk profile of the operation although showing distinctive signs of prosthetic loosening on both sides. The clinical und radiological follow up for one year confirmed the diagnosis of loosened prostheses and its progression. These cases were therefore evaluated as true positive.

One patient with a positive ${ }^{18} \mathrm{~F}$-Fluoride PET/CT finding for a loosening of the tibial component of the total knee arthroplasty has not been operated and showed no clinical symptoms in the follow up counting as false positive.
${ }^{18}$ F-Fluoride PET/CT correctly identified 18 out of 19 loosened total hip and knee arthroplasty components. In 6 out of 55 components without intraoperative or clinical signs of loosening we found a positive ${ }^{18} \mathrm{~F}$-Fluoride PET/ $\mathrm{CT}$ enhancement around the prosthesis counting as false positive. The others were correctly identified as not loosened in the scan.

On the basis of these data and considering every single prosthesis component (total 74, view Table 1) we found a sensitivity of $95.00 \%$, a specificity of 87.04 $\%$ and an accuracy of $89.19 \%$. These data include the clinically silent additional 11 prostheses (22 components).

When considering the respective endoprosthesis as one compound without distinguishing the femoral, tibial or acetabular component ( $\mathrm{n}=37$, view Table 1$)$, sensitivity lowers to $92.86 \%$ with a specificity of $86.96 \%$ (accuracy $=89.19 \%$ ). Since indication for revision surgery is given if at least one component of the prosthesis is being detected as loose, this perspective rather reflects clinical reality.

When considering only the painful and clinically suspicious prostheses (26, view Table 1, marked bold), which led to the patient undergo diagnostics, sensitivity is $92.31 \%$ and specificity lowers to $76.92 \%$ (accuracy = $84.62 \%)$.

\section{DISCUSSION}

The aim of this study was to evaluate the role of ${ }^{18} \mathrm{~F}$-Fluoride PET/CT in diagnosing periprosthetic loosening in total hip and knee arthroplasty.

M. Blau et al. were the first to use Fluorine-18 in bone imaging in 1962 [7], whereas Creutzig et al. presented a study of 31 hip prosthesis which he examined towards loosening and infection using planar ${ }^{18} \mathrm{~F}$-Fluoride scanning in 1976 [8]. Since then little research has been performed regarding this radiopharmaceutical and prosthesis loosening. Nevertheless, other impairments of bone metabolic activity have been very well investigated using ${ }^{18} \mathrm{~F}$-Fluoride PET, including renal osteodystrophy [9], osteoporosis [10], osseointegration of bone allografts [11], femoral head necrosis [12] and even bone malignancies [13].

In 2006 Sterner et al. showed in a study of 14 knee prostheses a sensitivity of $100 \%$ for diagnosing aseptic loosening with ${ }^{18} \mathrm{~F}$-Fluoride PET/CT, whereas specificity was very low with $56 \%$ and the relatively high rate of false positive results could not be explained by the authors [14]. Still, they were the first to present results of ${ }^{18} \mathrm{~F}$-Fluoride PET imaging in diagnosis of aseptic loosening. Compared to the above mentioned study our ${ }^{18} \mathrm{~F}$-Fluoride PET/CT data show both a high sensitivity and superior specificity.

The so far most investigated radionuclide procedure used for imaging joint arthroplasties is bone scintigraphy with Technetium-99m $\left({ }^{99 m} \mathrm{Tc}\right)$ labeled diphosphonates, usually methylene diphosphonate (MDP). No matter what 
Table 1: Included patients with total hip arthroplasty (THA) and total knee arthroplasty (TKA)

\begin{tabular}{|c|c|c|c|c|c|c|c|c|c|c|}
\hline Patient & Prosthesis & Sex & $\begin{array}{l}\text { Age } \\
\text { (y) }\end{array}$ & Joint & $\begin{array}{c}\text { Result of } \\
{ }^{18} \text { F-Fluoride } \\
\text { PET/CT }\end{array}$ & $\begin{array}{c}\text { Follow- } \\
\text { up }\end{array}$ & $\begin{array}{l}\text { Acetabular } \\
\text { Component }\end{array}$ & $\begin{array}{c}\text { Femoral } \\
\text { Component }\end{array}$ & $\begin{array}{c}\text { Tibial } \\
\text { Component }\end{array}$ & Comment \\
\hline \multirow[t]{2}{*}{1} & 1 & M & 69 & $\begin{array}{c}\text { THA } \\
\text { (right) }\end{array}$ & $\begin{array}{l}\text { Femoral } \\
\text { Loosening }\end{array}$ & Surgery & $\mathrm{TN}$ & ТP & - & $\begin{array}{c}\text { Exchange of } \\
\text { femoral component }\end{array}$ \\
\hline & 2 & & & $\begin{array}{l}\text { THA } \\
\text { (left) }\end{array}$ & $\begin{array}{c}\text { No } \\
\text { Loosening }\end{array}$ & Clinical & $\mathrm{TN}$ & $\mathrm{TN}$ & - & $\begin{array}{l}\text { No clinical } \\
\text { symptoms }\end{array}$ \\
\hline \multirow[t]{2}{*}{2} & 3 & $\mathrm{~F}$ & 72 & $\begin{array}{c}\text { THA } \\
\text { (right) }\end{array}$ & $\begin{array}{l}\text { Acetabular } \\
\text { loosening }\end{array}$ & Surgery & ТP & $\mathrm{TN}$ & - & $\begin{array}{l}\text { Exchange of } \\
\text { Prosthesis }\end{array}$ \\
\hline & 4 & & & $\begin{array}{l}\text { THA } \\
\text { (left) }\end{array}$ & No loosening & Clinical & $\mathrm{TN}$ & $\mathrm{TN}$ & - & $\begin{array}{l}\text { No clinical } \\
\text { symptoms }\end{array}$ \\
\hline \multirow[t]{2}{*}{3} & 5 & M & 83 & $\begin{array}{l}\text { THA } \\
\text { (left) }\end{array}$ & No loosening & Clinical & $\mathrm{TN}$ & $\mathrm{TN}$ & - & $\begin{array}{l}\text { Spine-related } \\
\text { symptoms }\end{array}$ \\
\hline & 6 & & & $\begin{array}{l}\text { THA } \\
\text { (left) }\end{array}$ & No loosening & Clinical & $\mathrm{TN}$ & $\mathrm{TN}$ & - & $\begin{array}{l}\text { No clinical } \\
\text { symptoms }\end{array}$ \\
\hline 4 & 7 & M & 68 & $\begin{array}{c}\text { TKA } \\
\text { (right) }\end{array}$ & $\begin{array}{c}\text { Femoral } \\
\text { loosening }\end{array}$ & Surgery & - & TP & $\mathrm{TN}$ & $\begin{array}{c}\text { Exchange of } \\
\text { femoral component }\end{array}$ \\
\hline 5 & 8 & M & 86 & $\begin{array}{l}\text { THA } \\
\text { (left) }\end{array}$ & $\begin{array}{l}\text { Femoral and } \\
\text { acetabular } \\
\text { loosening }\end{array}$ & Surgery & ТP & FP & - & $\begin{array}{l}\text { Exchange of } \\
\text { acetabular } \\
\text { component }\end{array}$ \\
\hline 6 & 9 & M & 73 & $\begin{array}{c}\text { THA } \\
\text { (right) }\end{array}$ & $\begin{array}{l}\text { Femoral and } \\
\text { acetabular } \\
\text { loosening }\end{array}$ & Surgery & TP & TP & - & $\begin{array}{l}\text { Exchange of } \\
\text { Prosthesis }\end{array}$ \\
\hline 7 & 10 & $\mathrm{~F}$ & 65 & $\begin{array}{c}\text { TKA } \\
\text { (right) }\end{array}$ & $\begin{array}{c}\text { Tibial } \\
\text { loosening }\end{array}$ & Clinical & - & $\mathrm{TN}$ & FP & $\begin{array}{c}\text { Regression of } \\
\text { symptoms after PT }\end{array}$ \\
\hline 8 & 11 & $\mathrm{~F}$ & 54 & $\begin{array}{l}\text { THA } \\
\text { (left) }\end{array}$ & $\begin{array}{l}\text { Acetabular } \\
\text { loosening }\end{array}$ & Surgery & ТP & $\mathrm{TN}$ & - & $\begin{array}{c}\text { Exchange of } \\
\text { Acetabular Cup }\end{array}$ \\
\hline 9 & 12 & $\mathrm{~F}$ & 55 & $\begin{array}{l}\text { THA } \\
\text { (left) }\end{array}$ & No loosening & Surgery & $\mathrm{TN}$ & $\mathrm{TN}$ & - & $\begin{array}{l}\text { Peri-articular } \\
\text { ossifications }\end{array}$ \\
\hline \multirow[t]{2}{*}{10} & 13 & $\mathrm{~F}$ & 80 & $\begin{array}{c}\text { THA } \\
\text { (right) }\end{array}$ & No loosening & Surgery & $\mathrm{TN}$ & $\mathrm{TN}$ & - & Tractus Gap \\
\hline & 14 & & & $\begin{array}{l}\text { TKA } \\
\text { (left) }\end{array}$ & No loosening & Clinical & - & $\mathrm{TN}$ & $\mathrm{TN}$ & $\begin{array}{l}\text { No clinical } \\
\text { symptoms }\end{array}$ \\
\hline \multirow[t]{2}{*}{11} & 15 & $\mathrm{~F}$ & 75 & $\begin{array}{l}\text { THA } \\
\text { (left) }\end{array}$ & No loosening & Surgery & $\mathrm{TN}$ & FN & - & $\begin{array}{c}\text { Exchange of } \\
\text { femoral component }\end{array}$ \\
\hline & 16 & & & $\begin{array}{c}\text { THA } \\
\text { (right) }\end{array}$ & No loosening & Clinical & $\mathrm{TN}$ & $\mathrm{TN}$ & - & $\begin{array}{l}\text { No clinical } \\
\text { symptoms }\end{array}$ \\
\hline \multirow[t]{2}{*}{12} & 17 & M & 64 & $\begin{array}{c}\text { THA } \\
\text { (right) }\end{array}$ & $\begin{array}{l}\text { Femoral and } \\
\text { acetabular } \\
\text { loosening }\end{array}$ & Surgery & FP & TP & - & $\begin{array}{c}\text { Exchange of } \\
\text { femoral component }\end{array}$ \\
\hline & 18 & & & $\begin{array}{l}\text { THA } \\
\text { (left) }\end{array}$ & No loosening & Clinical & $\mathrm{TN}$ & $\mathrm{TN}$ & - & $\begin{array}{l}\text { No clinical } \\
\text { symptoms }\end{array}$ \\
\hline 13 & 19 & $\mathrm{~F}$ & 92 & $\begin{array}{c}\text { TKA } \\
\text { (right) }\end{array}$ & $\begin{array}{l}\text { Femoral } \\
\text { and tibial } \\
\text { loosening }\end{array}$ & Clinical & - & ТP & TP & $\begin{array}{l}\text { Patient refused } \\
\text { surgery }\end{array}$ \\
\hline
\end{tabular}

(Continued) 


\begin{tabular}{|c|c|c|c|c|c|c|c|c|c|c|}
\hline Patient & Prosthesis & Sex & $\begin{array}{c}\text { Age } \\
(y)\end{array}$ & Joint & $\begin{array}{c}\text { Result of } \\
18 \text { F-Fluoride } \\
\text { PET/CT } \\
\end{array}$ & $\begin{array}{c}\text { Follow- } \\
\text { up }\end{array}$ & $\begin{array}{l}\text { Acetabular } \\
\text { Component }\end{array}$ & $\begin{array}{c}\text { Femoral } \\
\text { Component }\end{array}$ & $\begin{array}{c}\text { Tibial } \\
\text { Component }\end{array}$ & Comment \\
\hline & 20 & & & $\begin{array}{l}\text { TKA } \\
\text { (left) }\end{array}$ & $\begin{array}{l}\text { Femoral } \\
\text { and tibial } \\
\text { loosening }\end{array}$ & Clinical & - & $\mathrm{TP}$ & $\mathrm{TP}$ & $\begin{array}{l}\text { Patient refused } \\
\text { surgery }\end{array}$ \\
\hline 14 & 21 & M & 55 & $\begin{array}{l}\text { THA } \\
\text { (left) }\end{array}$ & $\begin{array}{l}\text { Femoral } \\
\text { loosening }\end{array}$ & Surgery & $\mathrm{TN}$ & TP & - & $\begin{array}{c}\text { Exchange of } \\
\text { femoral component }\end{array}$ \\
\hline 15 & 22 & $\mathrm{~F}$ & 67 & $\begin{array}{l}\text { TKA } \\
\text { (left) }\end{array}$ & $\begin{array}{c}\text { Femoral } \\
\text { and tibial } \\
\text { loosening }\end{array}$ & Surgery & - & TP & TP & $\begin{array}{l}\text { Exchange of } \\
\text { Prosthesis }\end{array}$ \\
\hline 16 & 23 & $\mathrm{~F}$ & 54 & $\begin{array}{l}\text { TKA } \\
\text { (left) }\end{array}$ & $\begin{array}{l}\text { Femoral } \\
\text { and tibial } \\
\text { loosening }\end{array}$ & Surgery & - & FP & TP & $\begin{array}{l}\text { Exchange of } \\
\text { Prosthesis }\end{array}$ \\
\hline 17 & 24 & $\mathrm{M}$ & 50 & $\begin{array}{c}\text { THA } \\
\text { (right) }\end{array}$ & No loosening & Clinical & $\mathrm{TN}$ & TN & - & $\begin{array}{c}\text { Regression of } \\
\text { symptoms after PT }\end{array}$ \\
\hline & 25 & & & $\begin{array}{l}\text { THA } \\
\text { (left) }\end{array}$ & No loosening & Clinical & $\mathrm{TN}$ & TN & - & $\begin{array}{l}\text { No clinical } \\
\text { symptoms }\end{array}$ \\
\hline 18 & 26 & $\mathrm{M}$ & 76 & $\begin{array}{l}\text { THA } \\
\text { (left) }\end{array}$ & No loosening & Clinical & $\mathrm{TN}$ & TN & - & $\begin{array}{c}\text { Regression of } \\
\text { symptoms after PT }\end{array}$ \\
\hline 19 & 27 & $\mathrm{M}$ & 63 & $\begin{array}{l}\text { THA } \\
\text { (left) }\end{array}$ & $\begin{array}{c}\text { Femoral and } \\
\text { acetabular } \\
\text { loosening }\end{array}$ & Clinical & ТP & ТP & - & $\begin{array}{l}\text { Patient refused } \\
\text { surgery }\end{array}$ \\
\hline 20 & 28 & M & 63 & $\begin{array}{l}\text { THA } \\
\text { (left) }\end{array}$ & $\begin{array}{c}\text { Femoral and } \\
\text { acetabular } \\
\text { loosening }\end{array}$ & Surgery & FP & ТP & - & $\begin{array}{c}\text { Exchange of } \\
\text { femoral component }\end{array}$ \\
\hline \multirow[t]{3}{*}{21} & 29 & $\mathrm{~F}$ & 74 & $\begin{array}{c}\text { TKA } \\
\text { (right) }\end{array}$ & $\begin{array}{l}\text { Femoral } \\
\text { loosening }\end{array}$ & Clinical & - & FP & $\mathrm{TN}$ & $\begin{array}{c}\text { Regression of } \\
\text { symptoms after PT }\end{array}$ \\
\hline & 30 & & & $\begin{array}{l}\text { TKA } \\
\text { (left) }\end{array}$ & No loosening & Clinical & - & $\mathrm{TN}$ & $\mathrm{TN}$ & $\begin{array}{l}\text { No clinical } \\
\text { symptoms }\end{array}$ \\
\hline & 31 & & & $\begin{array}{l}\text { THA } \\
\text { (left) }\end{array}$ & $\begin{array}{c}\text { No } \\
\text { Loosening }\end{array}$ & Clinical & $\mathrm{TN}$ & TN & - & $\begin{array}{l}\text { No clinical } \\
\text { symptoms }\end{array}$ \\
\hline 22 & 32 & $\mathrm{M}$ & 82 & $\begin{array}{c}\text { TKA } \\
\text { (right) }\end{array}$ & $\begin{array}{c}\text { No } \\
\text { Loosening }\end{array}$ & Surgery & - & TN & $\mathrm{TN}$ & Patellar surface \\
\hline 23 & 33 & $\mathrm{~F}$ & 53 & $\begin{array}{l}\text { TKA } \\
\text { (left) }\end{array}$ & No loosening & Surgery & - & TN & $\mathrm{TN}$ & Patellar surface \\
\hline 24 & 34 & M & 71 & $\begin{array}{c}\text { TKA } \\
\text { (right) }\end{array}$ & $\begin{array}{c}\text { No } \\
\text { Loosening }\end{array}$ & Surgery & - & $\mathrm{TN}$ & TN & Patellar surface \\
\hline 25 & 35 & $\mathrm{M}$ & 58 & $\begin{array}{l}\text { TKA } \\
\text { (left) }\end{array}$ & $\begin{array}{c}\text { Tibial } \\
\text { loosening }\end{array}$ & Surgery & - & TN & FP & Patellar surface \\
\hline \multirow[t]{2}{*}{26} & 36 & M & 66 & $\begin{array}{l}\text { THA } \\
\text { (left) }\end{array}$ & $\begin{array}{c}\text { No } \\
\text { Loosening }\end{array}$ & Clinical & $\mathrm{TN}$ & TN & - & $\begin{array}{l}\text { Soft tissue - related } \\
\text { symptoms }\end{array}$ \\
\hline & 37 & & & $\begin{array}{c}\text { THA } \\
\text { (right) }\end{array}$ & $\begin{array}{c}\text { No } \\
\text { Loosening }\end{array}$ & Clinical & $\mathrm{TN}$ & $\mathrm{TN}$ & - & $\begin{array}{l}\text { No clinical } \\
\text { symptoms }\end{array}$ \\
\hline
\end{tabular}

$\mathrm{TN}=$ True Negative, $\mathrm{TP}=$ True Positive, $\mathrm{FN}=$ False Negative, $\mathrm{FP}=$ False Positive, $\mathrm{PT}=$ Physiotherapy, bold $=$ clinically suspicious prostheses. 


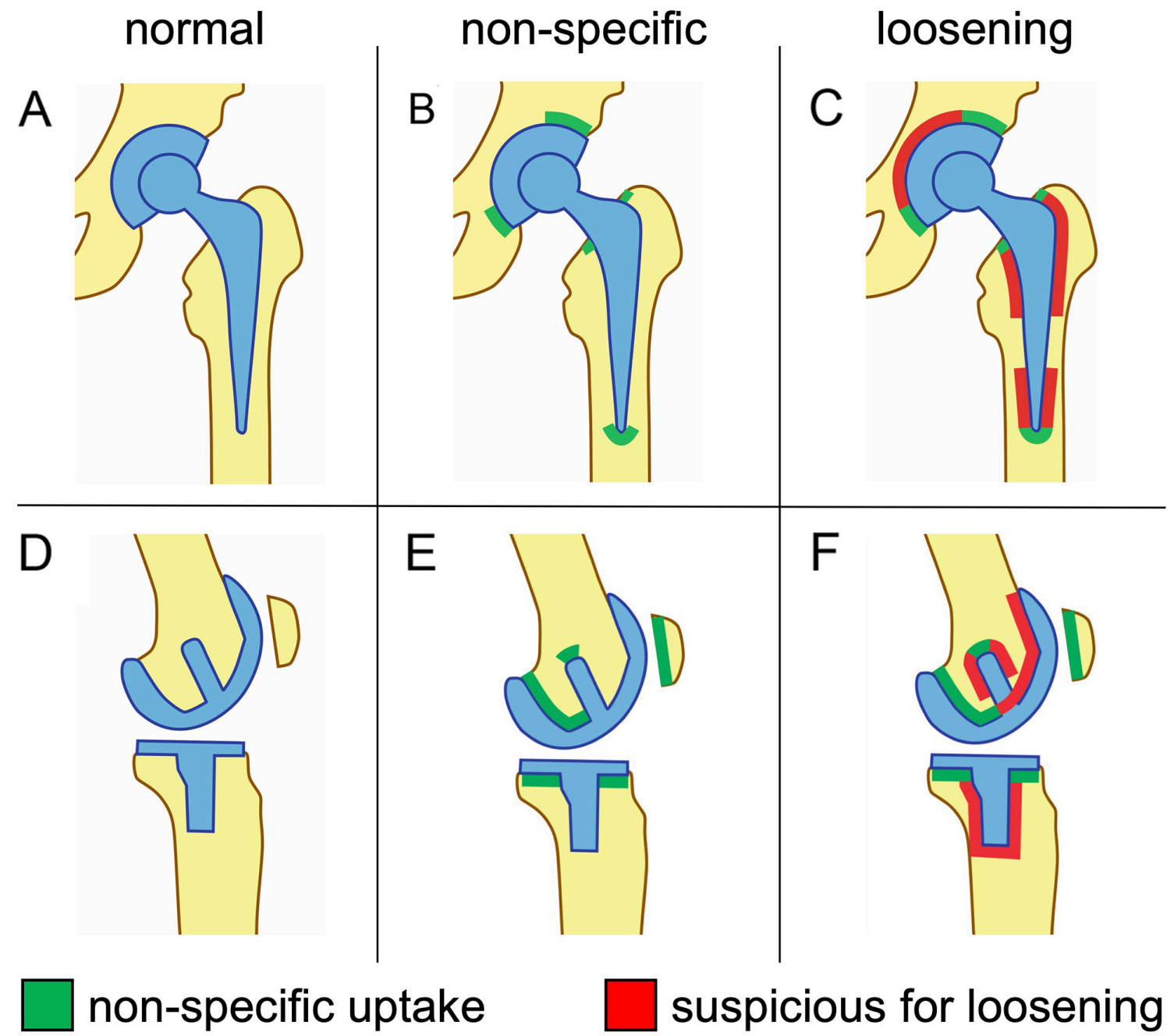

Figure 1: Schematic representation of ${ }^{18}$ F-Fluoride uptake patterns of total hip arthroplasties (A-C) and total knee arthroplasties (D-F) interpreted as non-specific (B, E) or suspicious for implant loosening $(\mathbf{C}, \mathbf{F})$.

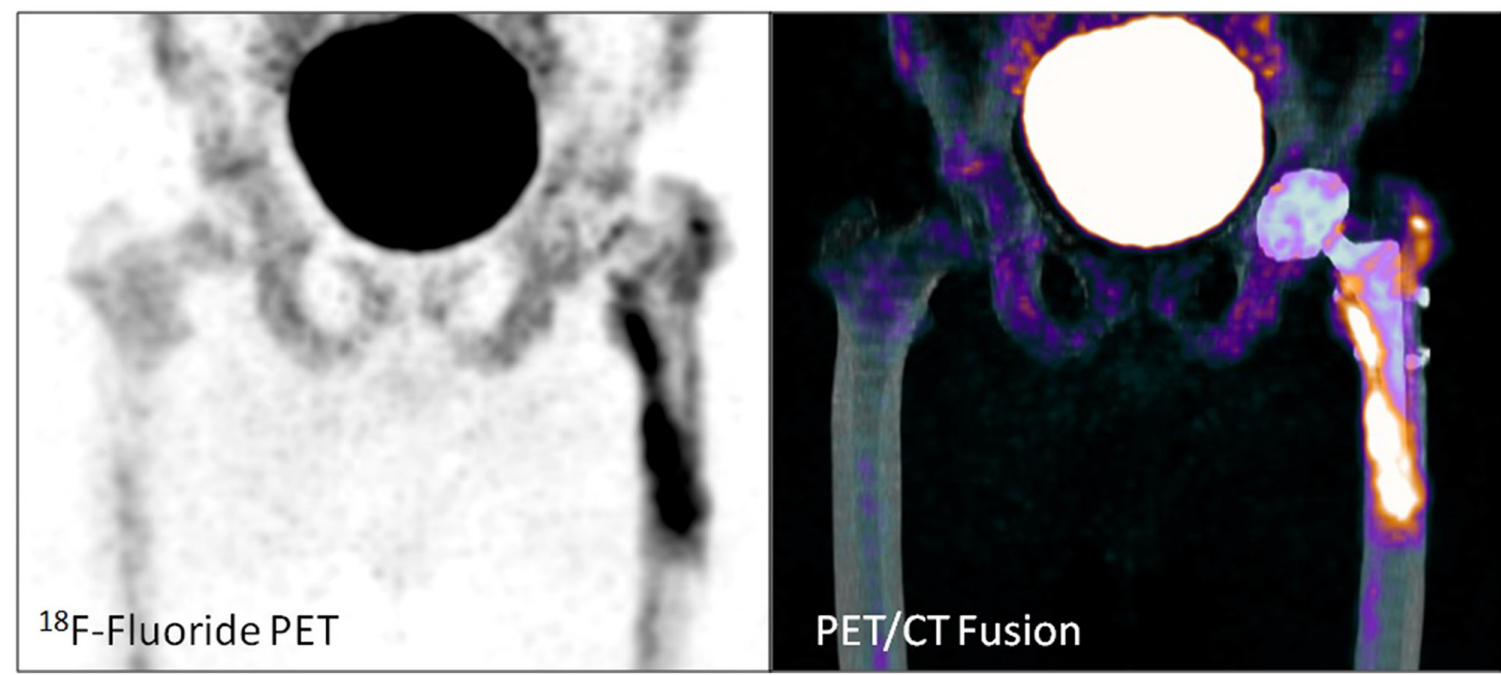

Figure 2: Total hip arthroplasty in a 55 year old male patient (patient number 14). 19 months after implantation. Loosening of the left femoral component (uptake along the stem including the tip). No signs of loosening of the left acetabular component. 

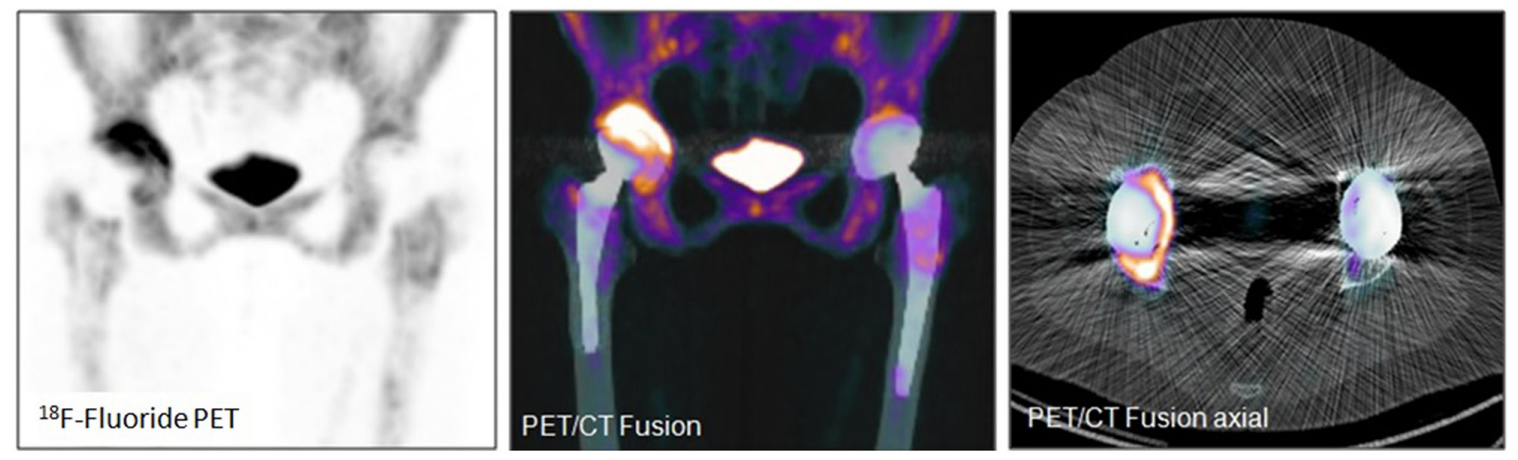

Figure 3: Total hip arthroplasty in a 72 year old female patient (patient number 2). 12.2 years after implantation. Loosening of the right acetabular component (circular uptake encompassing more than half of the acetabular component). No signs of loosening of the right femoral component, nor the left total hip arthroplasty.

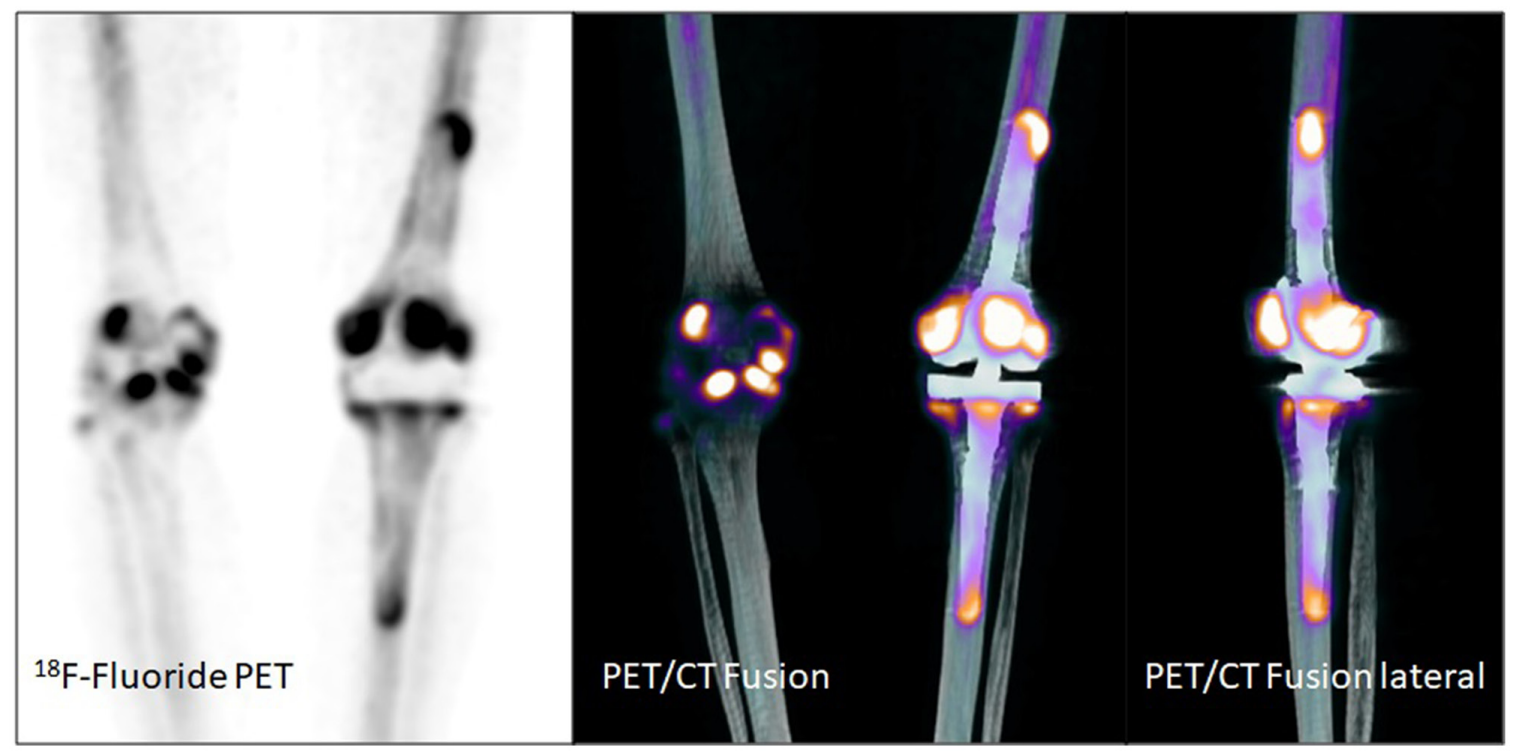

Figure 4: Total knee arthroplasty in a 67 year old female patient (patient number 15). 12 months after implantation. Suspicion of loosening of both components (femoral and tibial) of the total knee arthroplasty on the left side. Gonarthrosis on the right side.
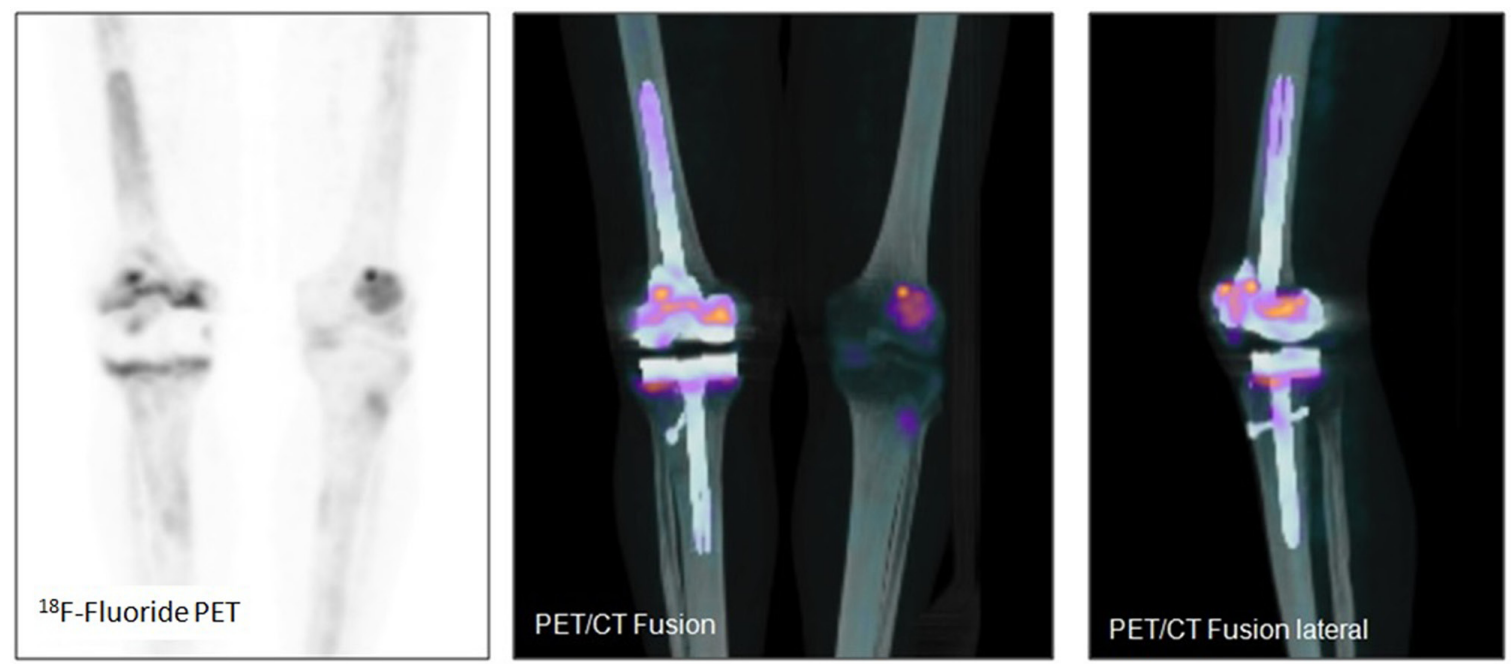

Figure 5: Total knee arthroplasty in an 82 year old male patient (patient number 22). 87 months after implantation. No signs of loosening, rather non-specific uptake along the weight bearing parts of the prosthesis. 
type of protocol or evaluation has been used, the accuracy of the method never exceeded $70 \%$, whereas specificity has always been reported low, mostly due to the limitations in spatial resolution and therefore evaluation of distinctive uptake patterns of the isotope [15-18]. ${ }^{18} \mathrm{~F}$-Fluoride PET/ CT however shows an excellent resolution enabling a more accurate observation of the pattern resulting in a higher specificity of the diagnosis.

In 2011 Choe et al. presented a comparatively large prospective trial towards differentiation between septic and aseptic loosening by using ${ }^{18} \mathrm{~F}$-Fluoride PET/ CT including 49 patients with 65 total hip arthroplasties. To our knowledge this represents the first prospective investigation in this regard [19] and the highest number of participants. The method involved measurement of the degree of ${ }^{18} \mathrm{~F}$-Fluoride uptake in periprosthetic tissue for septic and aseptic loosening. Our study did not include these types of measurements, but since differentiation between aseptic and septic loosening is key to an adapted treatment, further investigation in this matter is warranted.

Our and the above mentioned studies included patients whose prostheses have been implanted at least 1 year prior to ${ }^{18} \mathrm{~F}$-Fluoride PET/CT imaging. According to Creutzig et al. unspecific ${ }^{18} \mathrm{~F}$-Fluoride uptake especially around the acetabular component of total hip arthroplasty fades out about 9 months after implant surgery [8]. This is an argument in favor of using ${ }^{18} \mathrm{~F}$-Fluoride for this indication, considering that fluorodeoxyglucose $\left({ }^{18} \mathrm{~F}-\mathrm{FDG}\right)$, the most commonly used PET tracer, shows non-specific periprosthetic increased uptake for as long as several years, even in patients without evidence of infection or loosening [20]. However, also ${ }^{18} \mathrm{~F}$-Fluoride may show persisting non-specific uptake at the prosthesis/ bone interface of total hip and total knee arthroplasties [Son et al.], which necessitated the definition of nonspecific uptake patterns of ${ }^{18} \mathrm{~F}$-Fluoride in our study to reduce the number of false-positive results.

The emphasis in our study was put on the differentiation between loosened and not loosened prostheses, the differentiation between septic and aseptic loosening was not in the focus of our study. A quantitative evaluation has not been performed so far and serves as an limitation of the current study. However dynamic quantitative evalutation might give an insight into differentiation between septic and aseptic loosening. To address this topic, the evaluation of dynamic ${ }^{18} \mathrm{~F}$-Fluoride PET/CT scans with emphasis on an infection-related loosening will be part of further ongoing studies.

\section{PATIENTS AND METHODS}

\section{Patient population}

The study was approved of by the local institutional review board.
We included 26 patients in our retrospective study who suffered from painful total hip or total knee arthroplasties with radiological or clinical suspicion of peri-prosthetic loosening at least one year after implantation. Patients with prior oncologic disease have been excluded from the study. In the case of multiple inlaying prostheses without pain or clinical suspicion of loosening, the additional total knee and hip arthroplasties were also included into the study since the ${ }^{18} \mathrm{~F}$-Fluoride $\mathrm{PET} / \mathrm{CT}$ also produced images of these implants. All included patients were examined via ${ }^{18} \mathrm{~F}$-Fluoride PET/ CT between September 2014 and July 2017.

All patients were subject to routine clinical examination, laboratory and radiological studies. Data, such as development of pain and other clinical symptoms were gathered by chart review of medical records. No definite diagnosis had been established in any patient before the ${ }^{18}$ F-Fluoride PET/CT scan was performed. Revision operations were performed by experienced surgeons $1.4 \pm 0.2$ months after PET/CT scan. If no operation has been performed, the final evaluation and diagnosis was based on the long term clinical follow up of minimum 6 months (median: 11.3 months, range $6.0-29.7$ months). In these cases the patient either refused to undergo revision surgery and showed further progression of the radiological signs of loosening or experienced spontaneous regression of symptoms. All patients were examined with informed consent. Both components of the total hip (acetabular and femoral part) and total knee arthroplasty (femoral and tibial part) were independently evaluated as true positive or true negative according to the intraoperative findings or longterm clinical follow up. 9 patients had two, and one patient had 3 inlaying prostheses.

\section{PET scanning}

PET imaging was performed with a Biograph 2 PET/ CT scanner (Siemens Healthcare GmbH, Erlangen, Germany) with an axial field of view of $16.2 \mathrm{~cm}$ and a transversal field of view of $58.5 \mathrm{~cm} .{ }^{18} \mathrm{~F}$-Fluoride was injected intravenously at a mean activity of $292( \pm 24)$ MBq. Static emission scans of the legs were performed at a mean of $104( \pm 18) \min$ after injection (4 minutes emission time per bed position, 3D mode). PET images were reconstructed iteratively (4 iterations, 8 subsets, $256 \times 256$ matrix, $5 \mathrm{~mm}$ slice thickness). A low-dose CT $(16 \mathrm{mAs}, 130 \mathrm{kV})$ was acquired for attenuation correction and anatomical correlation.

\section{Image interpretation}

The scans were visually evaluated by an experienced nuclear medicine physician. The evaluation criteria were based on the pattern and location of the ${ }^{18} \mathrm{~F}$-Fluoride uptake at the prosthesis/bone interface or cement/bone interface.

The criteria we used in our current study to differentiate non-specific uptake of ${ }^{18} \mathrm{~F}$-Fluoride from 
patterns specific for loosening are in close correlation with uptake patterns described by Son et al., who previously examined postoperative ${ }^{18} \mathrm{~F}$-Fluoride uptake in asymptomatic patients up to 25 months after hip or knee arthroplasty [21]. Our criteria for non-specific uptake correspond to areas of the bone interface which showed intense uptake in more than $75 \%$ of asymptomatic patients in the study by Son et al. (Normal or no uptake see Figure $1(\mathrm{~A}, \mathrm{D}))$ Specifically, these are the cranial and caudomedial parts of the acetabular component and the distal tip of the femoral component of hip prostheses. Additionally, we categorized small areas of focal uptake in the proximal region of the femoral component as non-specific, as an increased frequency of persistent uptake of ${ }^{99 \mathrm{~m}} \mathrm{Tc}-\mathrm{HDP}$ has been observed in these areas in a previous study in asymptomatic patients with uncemented hip prostheses by Kim et al. [22], see Figure 1B. Regarding total knee arthroplasty, in correlation with the data by Son et al., uptake along the dorsal and caudal parts of the femoral sleds was rated as non-specific, as well as uptake solely in the horizontal surfaces in the proximal region of the tibial component. These criteria also closely match the methods used in the study by Sterner et al. for the detection of aseptic loosening of total knee arthroplasties by ${ }^{18} \mathrm{~F}$-Fluoride PET [14]. Additionally, we categorized uptake in the patellar region as non-specific, as well as small focal uptake at the tip of a pin/shaft of the femoral component, see Figure 1E.

Uptake exceeding these areas was interpreted as positive for loosening. Regarding hip prostheses, circular uptake at the interface encompassing more than half of the acetabular component was interpreted as positive for loosening, whereas for the femoral component, extended uptake around the trochanter regions or the distal parts of the shaft (exceeding the area of the tip) was rated positive (Figure 1C). With regard to total knee arthroplasties, uptake at the interface encompassing more than half of a condylar sled extending into the anterior parts, or extensive uptake around the pin/shaft of the femoral component was categorized as suspicious for loosening, as well as tracer accumulation around the pin/shaft of the tibial component (Figure 1F).

Examples of patient examinations are shown in Figures 2, 3, 4, 5.

\section{Data analysis}

The analogically recorded values out of the register were digitized and exported to MS Excel 2007 (Microsoft, Redmond, WA, USA) and SPSS 17 (IBM, Armonk, NY, USA) for further calculation. Subject of calculation by $2 \times 2$ table were sensitivity, specificity, accuracy.

\section{CONCLUSIONS}

The results of our study show that ${ }^{18} \mathrm{~F}$-Fluoride PET/ $\mathrm{CT}$ is a useful and promising technique in diagnosing periprosthetic loosening of total hip and knee arthroplasties. It demonstrates a very high sensitivity and specificity, not only compared to those of other imaging tools such as traditional bone scan, but also to other published trials in this regard. Moreover, it shows potential to completely replace traditional bone scan in the diagnostic pathway of periprosthetic loosening. Due to the promising findings of this retrospective analysis, further prospective studies are warranted with the recruitment of a higher number of patients to define the role and usage of ${ }^{18} \mathrm{~F}$-Fluoride $\mathrm{PET} / \mathrm{CT}$ in routine clinical practice. Future studies should also focus on the uptake kinetics of ${ }^{18} \mathrm{~F}$-Fluoride in periprosthetic joint infection to assess its value in distinguishing aseptic from septic loosening. Furthermore, the PET-scanning technique is also applicable with other tracers, such as ${ }^{68} \mathrm{Ga}$-zoledronate, which shows a slightly better availability than ${ }^{18} \mathrm{~F}$-Fluoride and is therefore subject of a current prospective study of the same group.

\section{ACKNOWLEDGMENTS}

The authors like to thank the technicians in the nuclear medicine department for performing the PET/CT scans.

\section{CONFLICTS OF INTEREST}

The authors state no conflicts of interest.

\section{REFERENCES}

1. Wimmer MD, Randau TM, Petersdorf S, Pagenstert GI, Weisskopf M, Wirtz DC, Gravius S. Evaluation of an interdisciplinary therapy algorithm in patients with prosthetic joint infections. Int Orthop. 2013; 37: 2271-8. https://doi.org/10.1007/s00264-013-1995-1.

2. Gravius S, Randau T, Wirtz DC. [What can be done when hip prostheses fail? : New trends in revision endoprosthetics]. [Article in German]. Orthopade. 2011; 40: 1084-94. https://doi.org/10.1007/s00132-011-1844-0.

3. Wirtz DC, Fischer H, Zilkens KW, Niethard FU, Marx R. [Optimizing the bone cement-implant interface by hydrolysis-resistant conditioning of the metal surface]. [Article in German]. Z Orthop Ihre Grenzgeb. 1999; 137: 447-51. https://doi.org/10.1055/s-2008-1037389.

4. Wirtz DC, Lelgemann B, Jungwirth F, Niethard FU, Marx R. [A new method to optimize the adhesion between bone cement and acetabular bone in total hip arthroplasty]. [Article in German]. Z Orthop Ihre Grenzgeb. 2003; 141: 209-16. https://doi.org/10.1055/s-2003-38661.

5. Even-Sapir E, Mishani E, Flusser G, Metser U. 18F-Fluoride positron emission tomography and positron emission tomography/computed tomography. Semin Nucl Med. 2007; 37: 462-9. https://doi.org/10.1053/j.semnuclmed.2007.07.002. 
6. Jadvar H, Desai B, Conti PS. Sodium 18F-fluoride PET/CT of bone, joint, and other disorders. Semin Nucl Med. 2015; 45 : 58-65. https://doi.org/10.1053/j.semnuclmed.2014.07.008.

7. Blau M, Nagler W, Bender MA. Fluorine-18: a new isotope for bone scanning. J Nucl Med. 1962; 3: 332-4.

8. Creutzig H. Bone imaging after total replacement arthroplasty of the hip joint. A follow-up with different radiopharmaceuticals. Eur J Nucl Med. 1976; 1: 177-80.

9. Messa C, Goodman WG, Hoh CK, Choi Y, Nissenson AR, Salusky IB, Phelps ME, Hawkins RA. Bone metabolic activity measured with positron emission tomography and $[18 \mathrm{~F}]$ fluoride ion in renal osteodystrophy: correlation with bone histomorphometry. J Clin Endocrinol Metab. 1993; 77: 949-55. https://doi.org/10.1210/jcem.77.4.8408470.

10. Berding G, Kirchhoff TD, Burchert W, von der Hoff J, Zeidler H, Hundeshagen H, Knapp WH. [18F]fluoride PET indicates reduced bone formation in severe glucocorticoidinduced osteoporosis. Nuklearmedizin. 1998; 37: 76-9.

11. Bernstein $\mathrm{P}$, Beuthien-Baumann B, Kotzerke J, Hofheinz F, Zessin J, Stiehler M, Gunther KP. Periacetabular bone metabolism following hip revision surgery. PET-based evaluation of allograft osteointegration. Nuklearmedizin. 2014; 53: 147-54. https://doi.org/10.3413/Nukmed-0607-13-06.

12. Schiepers C, Broos P, Miserez M, Bormans G, De Roo M. Measurement of skeletal flow with positron emission tomography and $18 \mathrm{~F}$-fluoride in femoral head osteonecrosis. Arch Orthop Trauma Surg. 1998; 118: 131-5.

13. Araz M, Aras G, Kucuk ON. The role of $18 \mathrm{~F}-\mathrm{NaF}$ PET/CT in metastatic bone disease. J Bone Oncol. 2015; 4: 92-7. https://doi.org/10.1016/j.jbo.2015.08.002.

14. Sterner T, Pink R, Freudenberg L, Jentzen T, Quitmann $\mathrm{H}$, Bockisch A, Loer F. The role of [18F]fluoride positron emission tomography in the early detection of aseptic loosening of total knee arthroplasty. Int J Surg. 2007; 5: 99-104. https://doi.org/10.1016/j.ijsu.2006.05.002.
15. Gallo J, Kaminek M, Myslivecek M, Zapletalova J, Spicka J. [Validity of bone scintigraphy for the diagnosis of periprosthetic complications in hydroxyapatite-coated total hip arthroplasty]. [Article in Czech]. Acta Chir Orthop Traumatol Cech. 2004; 71: 345-51.

16. Ashbrooke AB, Calvert PT. Bone scan appearances after uncemented hip replacement. J R Soc Med. 1990; 83: 768-9.

17. Rosenthall L, Lepanto L, Raymond F. Radiophosphate uptake in asymptomatic knee arthroplasty. J Nucl Med. 1987; 28: 1546-9.

18. Weiss PE, Mall JC, Hoffer PB, Murray WR, Rodrigo JJ, Genant HK. 99mTc-methylene diphosphonate bone imaging in the evaluation of total hip prostheses. Radiology. 1979; 133: 727-9. https://doi.org/10.1148/133.3.727.

19. Choe H, Inaba Y, Kobayashi N, Ike H, Aoki C, Shizukuishi $\mathrm{K}$, Iwamoto N, Yukizawa Y, Ishida T, Inoue T, Saito T. Use of $18 \mathrm{~F}$-fluoride PET to determine the appropriate tissue sampling region for improved sensitivity of tissue examinations in cases of suspected periprosthetic infection after total hip arthroplasty. Acta Orthop. 2011; 82: 427-32. https://doi.org/10.3109/17453674.2011.594232.

20. Zhuang H, Chacko TK, Hickeson M, Stevenson K, Feng Q, Ponzo F, Garino JP, Alavi A. Persistent non-specific FDG uptake on PET imaging following hip arthroplasty. Eur J Nucl Med Mol Imaging. 2002; 29: 1328-33. https://doi.org/10.1007/s00259-002-0886-2.

21. Son HJ, Jeong YJ, Yoon HJ, Wang L, Kim HJ, Park JH, Kang DY. Visual Pattern and Serial Quantitation of (18) F-Sodium Fluoride PET/CT in Asymptomatic Patients After Hip and Knee Arthroplasty. Nucl Med Mol Imaging. 2016; 50: 308-21. https://doi.org/10.1007/s13139-016-0430-0.

22. Kim HS, Suh JS, Han CD, Kim YH, Lee JD. Sequential Tc-99m MDP bone scans after cementless total hip arthroplasty in asymptomatic patients. Clin Nucl Med. 1997; 22: 6-12. 